\title{
ON THE SEMIGROUP STRUCTURE OF CONTINUA(1)
}

\author{
BY \\ ROBERT P. HUNTER
}

Let $S$ be a compact connected Hausdorff space. Suppose, moreover, that $S$ is a topological semigroup. A continuum $X$ is said to be aposyndetic, (Jones [8]), at a point $x$ with respect to a point $y$ if there is a subcontinuum $M$ and an open set $O$ such that $X-y \supset M \supset O \supset x$. It is well known, [8], that the set $T(p)$ is a continuum, where $T(p)$ denotes the set of points $x$ such that $X$ is not aposyndetic at $x$ with respect to $p$.

Our first section will be devoted to a study of the sets $T(p)$ in $S$. A number of nonaposyndetic analogues of Faucett's results, [6], will be developed.

The results on the sets $T(p)$ will be applied to continua, irreducible between two points, with $S^{2}=S$. It will be shown that if $S$ has a zero then $S$ is an arc. This includes a result of [15]. The case in which $S$ does not have a zero will be studied.

The results on the sets $T(p)$ and on irreducible continua will be applied to hereditarily unicoherent continua. It will follow, as a corollary, that if $S$ is one dimensional with unit and zero then it is arcwise connected.

It is with the utmost pleasure that we acknowledge our considerable indebtedness to Professor R. J. Koch for his advice, suggestions, and encouragement.

We now list some of the standard terms used in the study of topological semigroups. A left (right) ideal is a nonvoid subset $I$ such that $S I(I S)$ is a subset of $I$. The minimal ideal if it exists is denoted by $K$. It is known that if $S$ is compact then $K$ exists and is a retract of $S$. A subsemigroup $A$ is a subset such that $A^{2}$ is contained in $A$. By a clan we mean a compact connected semigroup with unit. If $e$ is an element such that $e^{2}=e$ then $e$ is called an idempotent. The set $E$ of all idempotents is closed. If $A$ is a subset then $J(A)=A+S A+A S+S A S$. The set $J_{p}$ is defined as the set of $x$ such that $J(p)=J(x)$. If $e$ is an idempotent then $H(e)$ denotes the maximal subgroup of $e$.

Again, we assume $S$ to be compact and connected.

1. Definition 1. The set $T(p)$ is said to be symmetric if for any $x$ in $T(p)$ it is true that $p$ is a point of $T(x)$.

The first part of the following theorem was proved in [13] under the assumption $S$ was a clan. For any set $M$, the symbol $M^{*}$ will denote the closure of $M$.

Received by the $\epsilon$ ditors September 22, 1958.

(1) This work was supported by the United States Air Force through the Air Force Office of Scientific Research and Development Command, under Contract AF 18(603)-89. 
THEOREM 1.1. If $S=E S+S E$ and $T(p)$ meets an ideal $I$ then $p$ is a point of I. If $T(p)$ is symmetric then $T(p)$ is contained in $I$.

Proof. Suppose on the contrary that $p$ is not in $I$. Let $x$ be a point in the common part of $I$ and $T(p)$. Let $D$ be an open set about $J(x)$ such that $D^{*}$ does not contain $p$. If $J$ denotes the sum of the ideals of $S$ contained in $D$ then $J$ is open by [16] and connected since $S=E S+S E$. But $J^{*}$ is a continuum containing $x$ within $J$, an open set. This is a contradiction.

The second statement follows from the first.

TheOREM 1.2. If $S=E S$ and $T(p)$ meets the left ideal $L$ then $p$ is a point of $L+K$. If $T(p)$ is symmetric then $T(p)$ is contained in $L+K$.

Proof. Similar to the above.

Theorem 1.3. If $S-T(p)=A+B$ mutually separate, $S=E S+S E$ and $K$ is a subset of $A$ then $J(p)$ is contained in $A^{*}$. If $T(p)$ is symmetric then $J(T(p)$ ) is contained in $A^{*}$.

Proof. If $J$ denotes the sum of the ideals contained in $A$ then $J$ is open (16). Since $J^{*}$ is an ideal it meets $T(p)$. By Theorem $1.1 p$ is in $J^{*}$ and the theorem follows.

It is easy to see that the restriction that $T(p)$ be symmetric for the second part of the above theorem is necessary.

Definition 1.2. An ideal is said to be prime if its complement is a semigroup.

Theorem 1.4. Suppose $T(p)$ is symmetric, $S=E S E$, and that $S-T(p)$ $=A+B$, mutually separate. If $A$ is a prime ideal then $T(p)$ is a group.

Proof. If $x$ and $y$ are two points of $T(p)$ then $x y$ is in $T(p)+B$ since $A$ is prime. Now $A^{*}$ contains $T(p)$ by Theorem 1.1 so that $x y$ is in $T(p)$. Thus $T(p)$ is a compact semigroup and, as such, contains an idempotent $e$. Using Theorem 1.2 we see that $e S$ and $S e$ contain $T(p)$ so that $e$ is a unit for $T(p)$. Furthermore $e$ is the only idempotent in $T(p)$, for if $f$ were another, one easily sees that $e=e f=f$, a contradiction. It now follows by [14] that $T(p)$ is a group.

The following notion will prove quite useful.

Definition 1.3 . The set $C$ is said to weakly cut the set $A$ from the set $B$ if $C$ meets every continuum which meets both $A$ and $B$. If $C$ is a point it is said to be a weak cut point.

ThEOREM 1.5. Suppose $T(p)$ is symmetric and weakly cuts the ideal $A$ from the set $B$. If $S=E S E$ and $(T(p))^{2}$ meets $T(p)$ then $(T(p)+B)^{2}$ does not meet $A$.

Proof. Suppose on the contrary that the points $x$ and $y$ are in $T(p)+B$ and that $x y$ is in $A$. Since $S x$ meets both $A$ and $B$ it meets, and hence contains, $T(p)$. Likewise $y S$ contains $T(p)$. Let $c$ and $d$ be points of $T(p)$ such 
that $c d$ is in $T(p)$. Now $c=s x$ and $d=y t$ so that $c d=(s x)(y t)=s(x y) t$ is a point of $A$ which is a contradiction.

The following is now immediate.

THeorem 1.5. Suppose $S=E S E$, the sct $T(p)$ is symmetric, $S-T(p)$ $=A+B$, mutually separate, and $A$ is an ideal. If $(T(p))^{2}$ meets $T(p)$ then $A$ is prime.

Definition 1.4. An ideal $I$ is semi-prime if $x^{2}$ is not in $I$ unless $x$ is a point of $I$.

Theorem 1.7. Suppose $S=E S E$ and that $S-T(p)=A+B$, mutually separate. If $A$ is a semi-prime ideal then $A$ is prime.

Proof. Since $p^{2}$ is not in $A$ and since $J(p)$ is contained in $A^{*}$ it follows that $p^{2}$ is a point of $T(p)$. Now suppose $x$ and $y$ are not in $A$ but $x y$ is. Since $S x$ meets $T(p)$ it follows that $p=s x$ for some $s$. Likewise $p=y t$. Now then, $p^{2}=(s x)(y t)=s(x y) t$ and hence is in $A$ since $A$ is an ideal. This is a contradiction.

Conditions in some of the previous theorems may be weakened or varied in accordance with the above.

THEOREM 1.8. Suppose $S=E S+S E$ and $p$ is in $S-K$. If $T(p)$ is symmetric it has vacuous interior.

Proof. We note that $T(p)$ does not meet $K$. Suppose that $T(p)$ contains an open set $O$. If $J$ denotes the sum of the ideals contained in $S-0^{*}$ then $J^{*}$ meets the boundary of $O$. But then $J^{*}$ contains $O$ which is impossible.

In both the above theorem and the following it is easy to see that the condition of symmetry is essential.

Theorem 1.9. Suppose $S=E S E$ and $p$ is in $S-K$. If $T(p)$ is symmetric and meets $H(e)$ then $T(p)$ is contained in $H(e)$.

Proof. Since $e S$ and $S e$ both contain $H(e)$ and consequently $T(p)$ it is clear that $T(p)$ is contained in $e S e$. If $x$ is any point of $T(p)$ then $S x$ contains $T(p)$ and hence contains $H(e)$. Likewise $x S$ contains $H(e)$. We conclude that $T(p)$ is contained in $H(e)$.

The following shows an important connection between a weak cut point $p$ and the set $T(p)$.

THEOREM 1.10. Let $X$ be a continuum and suppose $p$ weakly cuts a from $b$. If $T(p)$ contains neither $a$ nor $b$ then $T(p)$ separates $a$ from $b$.

Proof. Suppose on the contrary that $T(p)$ does not separate $a$ from $b$. For each point $x$ of $X-T(p)$ there is a subcontinuum $M$ and an open set $O$ such that $S-p \supset M \supset O \supset x$. The collection of all such open sets forms a covering of $X-T(p)$ and so there is a simple chain $O_{1}, O_{2}, \cdots, O_{n}$ with $O$ 
containing $a$ and $O_{n}$ containing $b$. Since each $O_{i}$ is contained in a continuum not containing $p$ it follows that there is a continuum containing $a$ and $b$ but not $p$. This is a contradiction.

Theorem 1.11. Suppose $S=E S+S E$ and that $A$ is the complement of a maximal prime ideal. If $V$ is an open subset about $A$ there is an open set $U$ such that $A \subset U \subset V$ and $S-U$ an ideal and hence connected.

Proof. For each point $x$ of $S-A$ there is, as in Theorem 1.1, a subcontinuum $M$ and an open set $O$ such that $S-A \supset M \supset O \supset x$. Hence if $V$ is open about $A$, the set $S-V$, since compact, is the sum of finitely many components. Since $A$ does not separate $S$, an easy argument, similar to Theorem 1.10 , shows the existence of the required set $U$.

Definition. A continuum is said to be the essential sum of a collection $G$ of continua if no element of $G$ is contained in the sum of the others. A continuum is said to be $n$-indecomposable if it is the essential sum of $n$ but not $n+1$ continua (Swingle, [20]).

We apply the set $T(p)$ to prove the following.

Theorem 1.12. If $S$ is $n$-indecomposable and $S=E S+S E$ then $S=K$. Hence, if $n>1$, multiplication must be (1) $x y=x$ for all $x, y$ or (2) $x y=y$ for all $x, y$.

Proof. It is clear that if we form $S / K$, assuming $K$ is proper, we see that there is an integer $r \leqq n$ such that $S / K$ is $r$-indecomposable. Hence we may, without loss, assume $S$ has a zero 0 . Swingle, [20], has shown that $S$ is the essential sum of $n$-indecomposable subcontinua $S_{1}, S_{2}, \cdots, S_{n}$. Let 0 be in $S_{1}$. Since $S=E S+S E$, it follows that for $p$, a point of $S_{1}$, the set $T(p)$ does not contain 0 . It is shown in (2) that this is impossible. Now an easy argument shows that $S$ cannot be the cartesian product of two nondegenerate continua. This together with the fact that $S=K$, shown above, and Corollary 1 of [16] implies the last statement of the theorem.

It is easy to see that if $S$ is 1 -indecomposable that any composant containing $K$ is an ideal and that it contains $E$.

2. Throughout this section we shall assume $S$ is a continuum irreducible between the points $a$ and $b$. That is, no proper subcontinuum contains $a$ and $b$.

We first examine the situation in which $S$ has a zero and prove the following. By an arc from $a$ to $b$, where $a$ and $b$ are points, we mean a continuum $X$ containing $a$ and $b$ with the property that any point of $X$, other than $a$ or $b$, separates $a$ from $b$. In other words $X$ is irreducibly connected between $a$ and $b$.

Theorem 2.1. If $S^{2}=S$ and $S$ has zero 0 then $S$ is an arc. Either $a$ or $b$ is idempotent. If $S$ has neither left nor right unit then both $a$ and $b$ are idempotent, $S-0=A+B$, mutually separate, and both $A^{*}$ and $B^{*}$ are abelian semigroups. If 0 does not separate $S$ then $S$ has a unit. 
Proof. Let us note first of all, that each set $T(p)$ is symmetric. This is quite easy to see since $S-T(p)$ has at most two components. Secondly, if $S$ has neither left nor right unit, then it follows, from the irreducibility as in [15] that $S=e S+S f$ and hence $S=e S e+f S f$ for $e$ and $f$ in $E$. Since, in this case, $a \neq 0 \neq b$ it follows that $T(0)=\{0\}$ separates $S$. In any case we see that $S=E S+S E$. A straightforward argument using Theorems 1.1 and 1.3 shows that if $T(x)$ meets $T(y)$ then $T(x)$ is contained in, or contains $T(y)$. This argument is similar to the one we now use to show that if $T(x)$ contains $T(y)$ then $T(x)=T(y)$. We suppose then that $T(x)$ properly contains $T(y)$ and we may suppose, without loss of generality, that $y$ is not in $T(a)+T(b)$. Then $S-T(y)=A+B$, mutually separate, with 0 an element of, say, $A$. By Theorem 1.2 the set $T(x)$ cannot meet $B$. Let $p$ be a point of $T(x)$ which is in $A$ but not $T(y)$. By symmetry, $T(p)$ does not contain $y$ and it follows that $S-T(p)=C+D$ separate with 0 in $C$ and $y$ in $D$. Since $x$ is in $T(p)$ we have a contradiction to Theorem 1.1 by means of Theorem 1.3. Hence the sets $T(p)$ are mutually exclusive, and an easy argument shows the collection, whose elements are the sets $T(p)$, to be upper semi-continuous. The hyperspace $G$ is seen to be an arc by Theorem 1.10. Let $e$ be an idempotent. We assert for any $T(x)$ in the interval, in $G$, from $T(0)$ to $T(e)$, that $T(x)=\{x\}$. We assume first that $T(x)$ contains no idempotent. Let $T(p)$ be the first element in the order from $T(x)$ to $T(e)$ such that $T(p)$ contains an idempotent $f$. It follows from [18] that in $f S f$ an $\operatorname{arc} A$ may be started at $f$. If $a$ is a point of $A$, by considering the locally connected continuum

$$
\left\{A+a A+a^{2} A+\cdots+a^{N} A\right\}
$$

for large enough $N$, and using the irreducibility of $S$, we see that $T(x)=\{x\}$. Now suppose that some $T(y)$, in the interval $T(0)$ to $T(e)$, is $T(g)$ for $g$ in $E$. In the interval from $T(0)$ to $T(g)$ one cannot have sets $T(x)$, containing no idempotent, arbitrarily close to $T(g)$. Since in $g S g$ there cannot be separating points arbitrarily close to $g$ unless $H(g)=g$, there is a subinterval $T(h)$ to $T(g)$ each element of which is a set containing an idempotent. However, by Theorem 1.9 each set $T(p)$ contains at most one idempotent. Hence there is a cross section at $e$ and by restricting the canonical mapping it follows that there is an arc from $T(h)$ to $T(g)$ and, finally, since there are not separating points close to $g$ in $g S g$, unless $H(g)=g$, we conclude that $T(g)=\{g\}$. It is now clear that there is, in $S$, an arc from 0 to any idempotent $e$. Since $S=E S+S E$ it follows that $S$ is an arc. The remaining statements are clear.

To simplify the discussion we use the notion of $C$-set as defined, for instance, in [21].

Definition 2.1. A subset $M$ of a space $X$ is called a $C$-set if any continuum meeting $M$ and $X-M$ must contain $M$.

The boundary of a set $A$ will always be denoted by $F(A)$. If there is a unique continuum irreducible from $c$ to $d$ it will be denoted by $[c, d]$. 
Lemma 2.1. Suppose $I$ is a closed subset of $S$, not separating $S$, such that $S^{\prime}$ (the space formed by shrinking I to a point) is an arc. If I has vacuous interior it is a $C$-set. If $I$ is an ideal and $S^{\prime}$ has a unit 1 and if $F(S-I)$ is nondegenerate then $S-I$ is an abelian semigroup and $F(S-I)$ is an abelian group.

Proof. The first conclusion is clear since each point of $S-I$ weakly cuts. To prove the second conclusion, let $x$ and $y$ be points of $S-I$ and suppose $x y$ is in $I$. Now $x[y, 1]$ is a locally connected continuum containing $x$ and meeting $I$. Since $S^{\prime}$ is an arc, it follows that $F(S-I)$ is degenerate. Since $S-I$ is abelian from [6] it follows that $(S-I)^{*}$ is abelian and its kernel is seen to be $F(S-I)$ which is then an abelian group.

In the remainder of this section, unless otherwise stated, we shall assume $K$ is nondegenerate.

Theorem 2.2. Suppose $K$ has vacuous interior. Then $K$ is a group and if it does not separate $S$ it is abelian and a $C$-set.

Proof. The case in which $K$ does not separate $S$ follows from Lemma 2.1.

We assume $K$ separates $S$ so that $S-K=A+B$, mutually separate. If $S$ has neither left nor right unit then from Theorem 2.1 we see that $a$ and $b$ are in $E$. If $F(A)$ is nondegenerate it is, by Lemma 2.1, a group. The same is true for $F(B)$. Clearly one or the other is nondegenerate. Since $K=F(A)$ $+F(B)$ it follows that $K$ is itself a group.

Let us suppose, now, that $S$ has a left unit $e$ which is in $A$. If $F(A)$ is degenerate then, letting $F(A)=k$, we see that the locally connected continuum $[k, e] b$ contains an arc from $b$ to $K$ and $K$ is degenerate. Hence $F(A)$ is nondegenerate and an abelian group by Lemma 2.1. We may suppose $K-F(A)$ is nonvacuous. We note that $F(B)$ contains $K-F(A)$ and is nondegenerate so that by Lemma $2.1 F(B)$ is a $C$-set in $B^{*}$. If $x$ is any point of $F(B)$, by continuity of multiplication, we see that $x S$ contains $F(B)$, as does $S x$. Further, since $F(A)$, a group, meets $x S$ it follows that $x S$ contains $K$. It follows from [14] that $K$ has a unit and consequently is a group.

Theorem 2.3. Suppose $K$ has a nonvacuous interior. One of the following must hold:

(i) each element of $K$ is a left zero.

(ii) each element of $K$ is a right zero.

(iii) $K$ is a group.

Proof. It follows from [15] that our theorem will be proved if we can show that $K$ is not the cartesian product of two nondegenerate continua. If $K$ were such a product it would be aposyndetic [8]. By the irreducibility of $S$, any point of the interior of $K$ would be a weak cut point of $K$. Such a point, by Theorem 1.10 , is a separating point of $K$. Since $K$ was a cartesian product this is impossible, and the theorem follows. 
THEOREM 2.4. If every element of $K$ is a left zero and $S$ has a left unit or has neither left nor right unit then $K$, and consequently $S$, is an arc.

Proof. If $S$ has left unit $e$ and $A$ is the component of $e$ in $S-K$ then $F(A)$, if nondegenerate, is a group by Lemma 2.1. Hence we may assume $F(A)$ $=\{k\}$ is degenerate. Taking $e=a$, and considering $[k, e] b$, the first part follows. In the second part we merely note that then $S=e S e+f S f$ and apply the first part.

If $K$ is composed of left zeros and $S$ has a right unit then any irreducible continuum may appear as $K$ as in Example 2.3.

THEOREм 2.5. If $K$ is a group with nonvacuous interior then it is indecomposable.

Proof. We assert that $K$ is irreducible between two points.

We suppose first that $K$ does not separate $S$ and take, using Theorem 2.1, $a$ as an idempotent and $b$ a point of $K$. Now the boundary of $S-K$ is either degenerate or a group by Lemma 2.1 and, in either case, has vacuous interior in $K$. It follows that $K$ is irreducible from $b$ to any point of $F(S-K)$.

We now suppose that $K$ separates $S$ and write $S-K=A+B$, mutually separate. If $S$ has neither left nor right unit then $F(A)$ is a group or degenerate and hence has no interior in $K$. The same is true for $F(B)$. It follows that $K$ is irreducible from $F(A)$ to $F(B)$. If $S$ has a left unit, say $a$, then $F(A)$ is nondegenerate and a group. (If not, we consider a translate by $b$ of the arc from $a$ to $K$.) Now $F(B)$ is a $C$-set in $B^{*}$. If $F(B)$ is degenerate it follows easily that $K$ is irreducible from $F(B)$ to $F(A)$. If $F(B)$ is nondegenerate it follows that if $x$ is in $B$ and $y$ is in $A$ the product $y x$ is in $B$, that is $A B$ is contained in $B$. By continuity of multiplication it follows that if $p$ is a point of $F(B)$ then $p F(A)$ contains $F(B)$. Hence $F(B)$ has no interior in $K$ and it follows that $K$ is irreducible from $F(A)$ to $F(B)$.

Since $K$ is irreducible between two points and is homogeneous it follows from [3] that $K$ is indecomposable.

We now list some examples.

EXAmple 2.1. It is shown in [15] that if $G$ is any compact, connected, separable, abelian group then there is a clan, with kernel $G$, irreducible from $G$ to the unit.

Throughout the following examples, I will denote the usual unit interval, $S \times T$ will denote the cartesian product with coordinatewise multiplication.

Example 2.2. Let $S$ be the clan of Example 2.1. Let $C$ be a two point semigroup. Form $S \times C$ and shrink each set $g \times C$ to a point for $g$ in $G$. That is, define $(s, c) R\left(s^{\prime}, c^{\prime}\right)$ if $s=c$ and $s^{\prime}=c^{\prime}$ or if $s=s^{\prime}$ is in $G$. The usual methods show that $S / R$ is a semigroup irreducible between two points. $S$ may be described as a continuum group with two spirals winding upon it.

EXAmple 2.3. Let $N$ be any continuum irreducible between two points $c$ and $d$. For $n$ and $m$ in $N$ define the product $n m$ to be $n$. The semigroup 
$(N \times\{0\})+(\{c\} \times I)+(\{d\} \times I)$ is irreducible between two points, has a right unit, and has $N$ as kernel.

EXAMPLE 2.4. In [6] there is described a clan $S$ irreducibly connected between two points $k$ and $e$. Its kernel is nondegenerate and does not separate.

First form $S \times I$. We note that $(\{k\} \times I)+(S \times\{0\})+(\{e\} \times I)$ is a nonabelian clan, is an arc, and is separated by its kernel.

Secondly we note that the semigroup $(\{k\} \times I)+(S \times\{0\})$ is an arc, has a nondegenerate kernel which separates, and has neither left nor right unit.

EXAmPLE 2.5. Let $S$ be, as in Example 2.1, irreducible from $G$ to $u$. Forming $S \times I$ we see that $(\{e\} \times I)+(S \times\{0\})+(\{u\} \times I)$ is a clan which is irreducible.

Suppose $G$ contains a subgroup $H$ with the properties needed for Example 2.1. Form $S \times I$ and from the cylinder $H \times I$ construct $T$, irreducible as in Example 2.1. We see that $T+(S \times\{0\})$ is irreducible and has neither left nor right unit. Its kernel has vacuous interior but is not a $C$-set.

Concerning the above example, it is easy to see that if $S-K=A+B$, mutually separate $S$ has a left unit $e$ in $A$ and $F(A)=K$, then $F(B)$ is either equal to $K$ or is degenerate.

EXAMPLE 2.6. Let $G$ be an indecomposable continuum which is a group. Forming $G \times I$ we note that $(G \times\{0\})+(\{e\} \times I)$, where $e$ is the unit of $G$, is irreducible.

3. A continuum is said to be hereditarily unicoherent if the common part of two subcontinua, which intersect, is a continuum. If there is a unique continuum irreducible from the point $c$ to the point $d$ it will be denoted by $[c, d]$. It is obvious that in an hereditarily unicoherent continuum the subcontinua irreducible between two points are unique.

THEOREM 3.1. If $S$ is hereditarily unicoherent and has a unit 1 and a zero 0 then $S$ is arcwise connected. Further, the arc $[0,1]$ is an abelian semigroup.

Proof. We shall show first that the continuum irreducible from 0 to 1 is a semigroup. Let $x$ and $y$ be points of $[0,1]$ and suppose that $x y$ is not an element of $[0,1]$. We assert first that $x$ is not an element of $[0, x y] \cap[0,1]$. For if $x$ were, the continuum $[0, x] y$ contains $[0, x y]$ which, in turn, contains $[0, x]$ since the irreducible continua are unique, and finally, $[0, x] y$ contains $[0, x]$ since $x y$ is not in $[0, x]$. This is impossible by [14]. Hence we may suppose both $x$ and $y$ are not points of $[0, x y] \cap[0,1]$. We now note that $\{[0, x y]+[0,1]\}-\{[0, x y] \cap[0,1]\}=A+B$, mutually separate, where $x y$ is an element of $A$ and $x$ is an element of $B$. Since the continuum $x[y, 1]$ contains $x y$ and $x$, and again by the uniqueness of the irreducible continua, we conclude that $x[y, 1]$ meets the continuum $x[y, 1] \cap[0,1]$. Let $z$ be a point in the common part of these continua. We note $z=x t$ for some $t$ in $[y, 1]$.

We now assert that $y$ is in $t S$. We suppose, on the contrary that $y$ is not in $t S$. It is then clear by Theorem 1.2 that $t$ is not in $T(y)$. We consider two cases: the first, when 1 is not in $T(y)$, the second, when 1 is in $T(y)$. In the 
first, we assert that either $y$ weakly cuts $t$ from 1 or that $y$ weakly cuts 0 from $t$. If neither of these held, there would be a continuum containing 0 and 1 but not $y$ which would be a contradiction to the uniqueness of the irreducible subcontinua. Now if $y$ weakly cuts 0 from $t$ it is immediate that $y$ is in $t S$. Hence we may suppose that $y$ weakly cuts $t$ from 1 . Since $t$ is not in $T(y)$, and 1 is not in $T(y)$, it follows from Theorem 1.10 that $S-T(y)=A+B$, mutually separate, with $t$ in $A$ and 1 in $B$. Now $T(y)+B$ is a continuum containing $y$ and 1 and consequently, $[y, 1]$. Since $t$ was in $[y, 1]$, this is manifestly impossible. Hence we may suppose that 1 is in $T(y)$. Since $T(y)$ then contains $[y, 1]$ we conclude that $t$ is in $T(y)$ which is a contradiction.

We now have shown that $y$ is an element of $t S$ so that $y=t s$ for some $s$. Now $x y=x(t s)=(x t) s$, so that $x y=z s$ with $z$ in $[0, x y] \cap[0,1]$. Finally, $[0, z] s$ contains $[0, z s]=[0, x y]$ which properly contains $[0, z]$ since $x y$ is not in $[0, z]$. Since $[0, z] s$ properly contains $[0, z]$ we have a contradiction to $[14]$. Hence $[0,1]$ is a semigroup and by Theorem 2.1 is an arc. By [6] we know that $[0,1]$ is abelian. If $c$ and $d$ are two points of $S$, the locally connected continuum $c[0,1]+d[0,1]$, which is hereditarily unicoherent, obviously contains an arc $[c, d]$. It has been shown, in the proof of Theorem 5 of [13], for instance, that a one-dimensional clan with zero is hereditarily unicoherent.

Corollary. Suppose $S^{2}=S$ and $S$ has a zero. If $S$ is hereditarily unicoherent it is arcwise connected. In particular, a one dimensional clan with zero is acrwise connected.

Proof. The condition $S^{2}=S$ implies that $S=S E S$ [16]. If $s$ is any point of $S$ then $s=x e y$ for some $e$ in $E$. Now the clan $e S e$, if one dimensional, is hereditarily unicoherent by [13]. Hence there is an arc $[0, e]$.By consideration of $x[0, e] y$ the theorem follows.

THEOREM 3.2. Suppose $S$ is arcwise connected and hereditarily unicoherent. If $S$ has a zero 0 and $x$ weakly cuts 0 from $y$ then $y$ is not in $S x$.

Proof. If $y=s x$, the continuum $s[0, x]$ properly contains $[0, y]$ in contradiction to [14].

THEOREM 3.3. If $S$ is arcwise connected, hereditarily unicoherent, and has a zero 0 , then $p[0, q]=[0, p q]$ and $[0, p][0, q]=[0, p q]$.

Proof. Clearly $p[0, q]$ contains $[0, p q]$. Suppose, for some $x$ in $[0, q]$, that $p x$ is not in $[0, p q]$. Now $p[x, q]$ contains $p x$ and $p q$. Let $z$ be the first point of $[p x, 0]$ in the order from $p x$ to 0 which is a point of $[0, p q]$. We then have $z=p y$ for some $y$ in $[x, q]$ so that $x=y s$ for some $s$. But then, $p x=p(y s)$ $=(p y) s=z s$ in contradiction to Theorem 3.2.

For the second conclusion we note first that $[0, p][0, q]$ contains $[0, p q]$ and we suppose that for some $x$ in $[0, p]$ and $y$ in $[0, q]$ the point $x y$ is not in 
$[0, p q]$. We note that $x[y, q]$ contains $[x y, x q]$ and that $x q$ is an element of $[0, p q]$ by the first part of this theorem. If $z$ is the first point of $[x y, x q]$ in $[0, p q]$ then $z=x t$ for $t$ in $[y, q]$. Since $y=t s$, we see that $x y=z s$ in contradiction to Theorem 3.2.

THEOREM 3.4. Let $S$ be hereditarily unicoherent. If $S=E S+S E$ and has a zero 0 then $x$ being in $T(p)$ implies $p$ is not in $T(x)$.

Proof. Obviously we may assume $x \neq 0 \neq p$. We assert that $[0, p] \cap T(p)$ $=p$. Let $y$ be any point in $[0, p] \cap T(p)$. It follows from Theorem 1.1 that $J(y)$ contains $p$ which, by the usual argument, is impossible. Now suppose for some $x$ in $T(p)$ that $p$ is in $T(x)$. Now $T(p)$ contains $[p, x]$. Since $[0, p]$ $\cap T(p)=p$ we conclude $[0, p]+[p, x]=[0, x]$ but by the first part, $T(x)$ $\cap[0, x]=x$. We conclude that $x=p$.

Definition 3.1. Let $S$ be arcwise connected and hereditarily unicoherent. By an endpoint we mean a point which separates no arc.

The following theorem implies that a one dimensional continuum with unit and zero, which is a subset of the plane, is accessible at each of its nonzero endpoints from its single complementary domain.

A problem, which we leave unsolved, is when such a semigroup is accessible at its zero.

Theorem 3.5. Suppose $S$ is hereditarily unicoherent and $S=E S+S E$. If $p$ is an endpoint and $p$ is not in $K$ then $S$ is semi-locally connected at $p$.

Proof. Since we may form the Rees quotient $S / K$, we shall assume $S$ has a zero 0 . Let $x$ be a point of $T(p)$. If $x$ is in $[0, p] \cap T(p)$ then $x=p$. If $x$ is not in $[0, p]$ then it follows that $p$ separates the arc $[0, x]$. Hence $T(p)=p$. An easy argument shows that if $V$ is an open set about $p$, there is an open set $U$ containing $p$ such that $U$ is a subset of $V$, and $S-U$ is connected.

The notion of limiting set, $\left(A_{\alpha} \rightarrow A\right)$, as used in the following, is the usual one as described, for example, in [12].

THEOREM 3.6. Suppose $S$ is hereditarily unicoherent having a left unit e and a zero 0 . If $\left\{A_{\alpha}\right\}$ is a collection of arcs each with 0 as an endpoint and $A_{\alpha} \rightarrow A$ then $A$ is an arc.

Proof. Let $a_{\alpha}$ be the nonzero endpoint of $A_{\alpha}$ and suppose $a_{\alpha} \rightarrow a$. By continuity, $[0, e] a_{\alpha} \rightarrow[0, e] a$. By Theorem 3.3 , this implies $\left[0, a_{\alpha}\right] \rightarrow[0, a]$.

This theorem also follows from Theorem 3.4, but not in so direct a fashion.

Theorem 3.7. Suppose $S$ is hereditarily unicoherent, has a zero 0 , and a left unit. If one defines $x \leqq y$ if either $x$ weakly cuts 0 from $y$ or $x=0$, then $\leqq i$ an order dense continuous partial order.

Proof. It is clear that $\leqq$ is an order dense partial order. To show continuity, suppose $a$ 
$V$ about $a$ and $b$ such that $u \$ v$ and $v \underline{u}$ for $u$ in $U$ and $v$ in $V$. Suppose on the contrary that there exist arbitrarily small $U_{\alpha}$ and $V_{\alpha}$ such that $u_{\alpha} \leqq v_{\alpha}$ for $u_{\alpha}$ in $U_{\alpha}$ and $v_{\alpha}$ in $V_{\alpha}$. It then follows that $\left[0, v_{\alpha}\right]=\left[0, u_{\alpha}\right]+\left[u_{\alpha}, v_{\alpha}\right]$. Now the limiting set of $\left\{\left[0, v_{\alpha}\right]\right\}$, (or some subcollection), contains $0, a$, and $b$, and, since it is an arc from the previous theorem, we conclude that 0 weakly cuts between $a$ and $b$. Now $S$ is locally connected at 0 so that we may find an open set $D$ about 0 such that $D^{*}$ is a continuum. An easy argument shows that for some $\beta$, a subarc of $\left[0, v_{\beta}\right]$ has its endpoints in $D^{*}$ but is not a subset of $D^{*}$. Since this is impossible the proof is complete.

Definition 3.2. By a maximal arc we mean one which is not a proper subset of any other arc.

It can be shown, using standard techniques, that in an arcwise connected hereditarily unicoherent continuum, any arc can be extended to a maximal arc. The situation is somewhat easier with a semigroup as the following shows.

THEOREM 3.8. If $S$ is hereditarily unicoherent with a left unit e and a zero 0 then $S$ is arcwise connected and every arc is contained in a maximal arc.

Proof. Arcwise connectedness follows from Theorem 3.3. Let $[0, a]$ be an arc. Let $Q$ be the collection of all arcs of the form $\left[0, x_{\alpha}\right], \alpha$ in $A$, where $\left[0, x_{\alpha}\right]$ contains $[0, a]$. Let $T$ be a maximal tower in $Q$ and let $L$ be the closure of the union of the elements of $T$. Define $b_{\alpha} \leqq b_{\beta}$ if $b_{\alpha}$ weakly cuts 0 from $b_{\beta}$ if and only if $\left[0, b_{\alpha}\right]$ is contained in $\left[0, b_{\beta}\right]$. Note that $(A, \leqq)$ is a directed set. Let $b$ be a cluster point of $\left\{b_{\beta}\right\}$. By [14] and Theorem 3.3, we see that $L=\left(U\left[0, b_{\beta}\right]\right)^{*}=\left(U[0, e] b_{\beta}\right)^{*}=[0, e] b=[0, b]$ and the theorem follows.

Concerning the existence of weak cut points we have the following.

Theorem 3.9. Suppose $S$ is one dimensional and has a unit. If $S$ has no weak cut point then $S$ is a simple closed curve.

Proof. If $K$ is not proper form $S / K$. We know $S / K$ is arcwise connected and hereditarily unicoherent. Let 0 be the zero of $S / K$. Since $S / K-0$ and $S-K$ are homeomorphic it follows that $S$ has a weak cutpoint. Hence we know that $S=K$ and consequently $S$ is a topological group. Now $S$ is certainly not indecomposable for every point of an indecomposable continuum is a weak cutpoint. Since $S$ is a decomposable, one dimensional, compact, connected, topological group it is a simple closed curve.

THEOREM 3.10. Suppose $S$ is arcwise connected, hereditarily unicoherent and equal to $E S+S E$. If $A$ is the complement of a maximal proper ideal $M$ then every point of $A$ is an endpoint and $A$ is totally disconnected.

Proof. We may assume $S$ has a zero 0 . Let $a$ be in $A$ and assume $a$ is not an endpoint. Then $[0, a]$ is a proper subset of some $[0, t]$. Now $J(a) \cap[a, t]$ $=a$. Since $M+J(a)=S$ we conclude $[a, t]-a$ is a subset of $M$. Since $J(t)$ is contained in $M$ we see that $a$ is in $M$ which is impossible. 
Theorem 3.11. Suppose $S$ is arcwise connected, hereditarily unicoherent and has $a$ zero. If the endpoints are idempotent and commute, one with another, then $S$ is abelian.

Proof. If $r$ and $s$ are any two points of $S$ then $r$ is in $[0, e]$ and $s$ is in $[0, f]$ for some idempotents $e$ and $f$. We note, from Theorem 3.3 , that $[0, e][0, f]$ $=[0, e f]=[0, f e]=[0, f][0, e]$ and that all of these contain $r s$ and $s r$.

Now $r s=(r e)(f s)=r(f e) s=(r f)(e s)$. Both $r f$ and $e s$ are points of $[0, e f]$ which is abelian by Theorem 3.1. Hence $(r f)(e s)=e(s r) f$. We now assert that $e(s r) f=s r$. Ordering $[0, e f]$ from 0 to $e f$, we cannot have $e(s r) f<s r$, using Theorem 3.2, and were we to have $e(s r) f<s r$, then $f(e(s r) f) e=(f e)(s r)(f e)=s r$, again a contradiction to Theorem 3.2. Hence, $S$ is abelian.

Corollary (FAUCETT). If $S$ is irreducibly connected between two idempotents which commute and has a zero then $S$ is abelian.

THEOREM 3.12. Suppose $S$ is hereditarily unicoherent with unit 1 and zero 0. If every endpoint is an element of $H(1)$ then $H(1)$ and $[0,1]$ commute elementwise. Hence if $H(1)$ is abelian so is $S$.

Proof. Using Theorems 3.1 and 3.3, the argument of [7] suffices.

We note that if $S$ is hereditarily unicoherent and has a unit and a zero it has the fixed point property. This is, certainly in the metric case, well known [1]. It also follows from Theorem 3.7 and [22].

THEOREM 3.13. Let $S$ be one dimensional with unit 1. If $S$ is not arcwise connected then $K$ is a group.

Proof. Since $K$ is one dimensional it is not the cartesian product of two nondegenerate continua. Hence, [15], every element of $K$ is a left (right) zero or $K$ is a group. The continuum $M$, irreducible about $K+\{1\}$, is a semigroup by Theorem 3.1. Let $N=M-K$, and let $F(N)$ be the boundary of $N$ in $N^{*}$. Since any point of $N$ weakly cuts $K$ from 1 it follows that $F(N)$ is a $C$-set in $N^{*}$ and hence by Lemma 2.1 , is a group if nondegenerate. Hence we may suppose $F(N)=\{k\}$ is degenerate. By considering translates of $[k, 1]$ the theorem follows.

By taking $C$ as the Cantor set under " $\min$ " and $G$ as the circle group in Example 2.2 the result is a totally nonaposyndetic clan which is one dimensional and has no separating point.

Let $C$ be the Cantor set under "min" and $S$ be a clan which is a triod with zero endpoint. Form $S \times C$ and shrink $\{0\}+C$ to a point. The resulting one dimensional clan with zero is not a subset of the plane.

\section{REFERENCES}

1. K. Borsuk, A theorem on fixed points, Bull. Acad. Polon. Sci. II, no. I (1954) pp. 17-20.

2. C. E. Burgess, Continua and their complementary domains in the plane, Duke Math. J. vol. 19 (1952) pp. 223-230. 
3. - Some theorems on N-homogeneous continua, Proc. Amer. Math. Soc. vol. 5 (1954) pp. 136-143.

4. A. H. Clifford, Semigroups containing minimal ideals, Amer. J. Math. vol. 70 (1948) pp. 521-526.

5. H. Cohen, A cohomological definition of dimension for locally compact Hausdorff spaces, Duke Math. J. vol. 21 (1954) pp. 209-224.

6. W. M. Faucett, Compact semigroups irreducibly connected between two idempotents, Proc. Amer. Math. Soc. vol. 6 (1955) pp. 741-747.

7. - Topological semigroups and continua with cut points, Proc. Amer. Math. Soc. vol. 6 (1955) pp. 748-756.

8. F. B. Jones, Aposyndetic continua and certain boundary value problems, Amer. J. Math. vol. 67 (1941) pp. 545-553.

9. $\longrightarrow$, Concerning aposyndetic and non-aposyndetic continua, Bull. Amer. Math. Soc. vol. 58 (1952) pp. 137-151. 413.

10. — Concerning non-aposyndetic continua, Amer. J. Math. vol. 70 (1948) pp. 403-

11. On a certain type of homogeneous plane continuum, Proc. Amer. Math. Soc. vol. 6 (1955) pp. 735-740.

12. R. J. Koch, Arcs in partially ordered spaces, Ann. of Math., to appear.

13. - Note on weak cut points in clans, Duke Math. J., vol. 24 (1957) pp. 611-616.

14. - On monothetic semigroups, Proc. Amer. Math. Soc. vol. 8 (1957) pp. 397-401.

15. R. J. Koch and A. D. Wallace, Admissibility of semigroup structures on continua, Trans. Amer. Math. Soc. vol. 88 (1958) pp. 277-287. 685.

16. - Maximal ideals in compact semigroups, Duke Math. J. vol. 21 (1954) pp. 681-

17. L. F. McAuley, On the decomposition of continua into aposyndetic continua, Trans. Amer. Math. Soc. vol. 66 (1956) pp. 74-91.

18. P. S. Mostert and A. L. Shields, On the structure of semigroups on a compact manifold with boundary, Ann. of Math. vol. 65 (1957) pp. 117-143.

19. P. M. Swingle, End sets of continua irreducible between two points, Fund. Math. vol. 17 (1931) pp. 40-76. 658.

20. - Generalized indecomposable continua, Amer. J. Math. vol. 52 (1930) pp. 647-

21. A. D. Wallace, The position of $C$-sets in semigroups, Proc. Amer. Math. Soc. vol. 6 (1955) pp. 639-642.

22. L. E. Ward, Mobs, trees and fixed points, Proc. Amer. Math. Soc. vol. 8 (1957) pp. 798804.

Louisiana State University, Baton Rouge, La. 